\title{
Versionamento de Recursos em Repositórios de Objetos de Aprendizagem: uma revisão sistemática
}

\author{
José Wallison Félix da Silva - PPGCC-IFCE, wallison.felix@ppgcc.ifce.edu.br \\ Cidcley Teixeira de Souza - PPGCC-IFCE, cidcley@ifce.edu.br
}

\begin{abstract}
Resumo. Reúso é uma das principais características dos Objetos de Aprendizagem (OAs). Para reutilizar estes recursos, às vezes é necessário realizar modificações a fim de adequá-los a novos contextos de uso. Estas alterações resultam em novas versões do OA. Por isso, recomenda-se que os Repositórios de Objetos de Aprendizagem (ROAs) possuam estratégias para o gerenciamento de diferentes versões dos recursos. Este artigo descreve uma Revisão Sistemática $(R S)$ realizada com o objetivo de identificar como estes repositórios realizam o versionamento do seu conteúdo, de modo a auxiliar equipes responsáveis pela modelagem e implementação destas bases em relação a tomada de decisão sobre como desenvolver o Controle de Versão de OAs. Para a obtenção de publicações que detalhem a execução do gerenciamento de versões em ROAs, bases digitais de publicações relevantes na área da Engenharia de Software foram consultadas. As publicações retornadas foram avaliadas por meio de um Protocolo de Revisão (PR), a fim de garantir a seleção apenas de trabalhos compatíveis com os objetivos da revisão. Esta RS permitiu concluir que o versionamento em ROAs é basicamente realizado de duas formas: alterando padrões de metadados utilizados para descrever OAs ou definindo modelagens de dados que permitam estabelecer relação entre as versões.
\end{abstract}

Palavras-chave: objetos de aprendizagem, repositório de objeto de aprendizagem, controle de versão, versionamento, revisão sistemática.

\section{Versioning of Resources in Learning Objects Repositories: a systematic review}

\begin{abstract}
Reusability is one of the most important features in Learning Objects (LOs). Sometimes these resources demand modifications in order to reuse in others use contexts. This adaptation process results in new versions of a resource. Therefore, it is recommended that Learning Objects Repositories (LORs) have some strategy to manage these versions. This paper describes a Systematic Review (SR) to detect how this type of repository performs the versioning of content. The results aims to aid teams responsible for modeling and developing these repositories in a decision about how to implement versioning of LOs. Digital bases of relevant publications on Software Engineering were queried in order to obtain publications describing version management in LORs. Found papers were evaluated by a Review Protocol (RP) to ensure selection just of publications compatible with the SR objective. This SR concludes Version Control in LOR is realized in two ways: changing metadata standards used to describe LOs or defining data models to allow relationship establishment between versions.
\end{abstract}

Keywords: learning objects, learning object repository, version control, versioning, systematic review. 


\section{Introdução}

A Sociedade da Informação e do Conhecimento baseia-se nos meios de comunicação oferecidos pelas Tecnologias da Informação e da Comunicação (TICs) (Tarouco, 2014), e, segundo Castells (1999), este é o modelo de sociedade para o qual caminhamos. Resultado disso na educação é a notada intensificação do uso de TICs no ambiente educacional (Schmitt, 2013). Neste contexto, os Objetos de Aprendizagem (OAs) se destacam como uma vantajosa ferramenta para o ensino-aprendizagem, pois permitem diversificar a abordagem de conteúdos e têm a reusabilidade como uma de suas principais características (Wiley, 2000). OAs são, conforme o difundido conceito de Wiley (2000), quaisquer recursos digitais que possam ser reutilizados como suporte à aprendizagem.

De acordo com o que é evidenciado em sua definição, o reúso é uma propriedade central dos OAs. Para facilitar o reúso destes recursos, eles devem ser compartilhados por meio de Repositórios de Objeto de Aprendizagem (ROAs), sistemas que facilitam o armazenamento, localização e recuperação de OAs (Tzikopoulos, Manouselis, Vuorikari, 2009). Neles os objetos são descritos através de metadados que são utilizados para a indexação do conteúdo (Higgs, Meredith, Hand, 2003).

A reutilização desses recursos acarreta em vários benefícios para professores, alunos e instituições (CISCO Systems, 2003). Além disso, gera economia e minimiza esforços, já que a produção de OAs pode resultar em altos custos, financeiros e temporais. Portanto, o ideal é que as instituições de ensino que produzem estes materiais também os compartilhem (Rensing, 2005; Schmitt, 2013). Contudo, há uma série de fatores que limitam a reusabilidade desses recursos (Tarouco, Silva, Grando, 2011).

Os Objetos de Aprendizagem às vezes precisam ter o seu conteúdo e/ou layout alterados para que sejam reutilizados. Isto ocorre para adaptá-los a novos contextos de uso (Schmitt, 2013). Em relação a essa readequação, Lehmann et al. (2008) destacam que uma das fases do ciclo de vida de um OA é justamente a Reautoria, fase na qual são executadas operações de adição, exclusão, modificação e/ou reordenamento de conteúdo. Estas alterações resultam na criação de novas versões dos recursos. Contudo, há um risco em potencial relacionado à modificação de OAs, que é o fato de a mudança impactar os seus demais utilizadores. Para mitigar este risco, versões anteriores devem ser mantidas (Tate, Hoshek, 2009). E ainda deve haver uma estratégia para controlar e gerenciar as várias versões existentes, mantendo o relacionamento entre elas, registrando as alterações realizadas e por quem e quando foram executadas (Farrell, 2010).

O efetivo reúso e as consequentes adaptações de OAs contidos em um ROA só podem ocorrer com uma estratégia de versionamento que dê suporte às várias versões dos recursos (Theilmann, Altenhofen, 2003). Os ROAs devem oferecer, como parte das suas funcionalidades para a gerência do repositório, uma estratégia de Controle de Versão (Higgs, Meredith, Hand, 2003). Sendo, inclusive, essa característica um dos fatores críticos para o sucesso deste tipo de base de dados, conforme afirma Sridharan, Deng e Corbitt (2010).

Os padrões de metadados Learning Object Metadata (LOM) e Dublin Core (DC), comumente utilizados para descrever OAs, dispõem de campos destinados ao versionamento de recursos. Mas gerenciar versões utilizando pura e simplesmente estes padrões, conforme são definidos, não é a forma mais adequada, pois não é possível registrar as alterações realizadas em um formato que sejam facilmente interpretadas por sistemas informatizados, de modo a permitir, por exemplo, a identificação automática das 
diferenças e o estabelecimento de relação entre as diversas versões do recurso (Brooks, 2005; Libbrecht, Books, 2006).

Portanto, considerando a importância de se ter uma estratégia de versionamento de recursos nos ROAs e que os padrões de metadados mais comumente utilizados não são suficientes para atender esta necessidade, decidiu-se realizar uma Revisão Sistemática (RS) da literatura a fim de identificar as estratégias utilizadas para o Controle de Versão de Recursos Educacionais Digitais (REDs) nestes repositórios. E, consequentemente, levantar conteúdo que auxilie, bem como inspire, equipes responsáveis pela modelagem e implementação de ROAs durante o processo de decisão sobre como realizar o versionamento de recursos.

Na seção a seguir, Revisão Sistemática, é apresentado o conceito de RS e o porquê de se utilizá-la, além de ser detalhado o Protocolo de Revisão (PR) adotado neste trabalho e apresentados os resultados oriundos da execução do protocolo. A terceira seção é destinada às informações extraídas das publicações selecionadas por meio do PR. Por fim, uma conclusão da RS é apresentada.

\section{Revisão Sistemática}

Revisão Sistemática da literatura é um meio de identificar, avaliar e interpretar todos os estudos relevantes relacionados a uma pergunta de pesquisa, ao tópico de uma área ou a um fenômeno de interesse (Kitchenham, 2004). Para facilitar o planejamento e a execução de revisões sistemáticas na área da Engenharia de Software, Biolchini et al. (2005) desenvolveram um template de Protocolo de Revisão que busca guiar o pesquisador ao longo de cada passo da Revisão Sistemática, definindo claramente o conteúdo de cada seção do protocolo. Este template foi baseado nos protocolos desenvolvidos para a área da Medicina e nas diretrizes propostas por Kitchenham (2004). Ele será utilizado como guia nesta RS. Basicamente, divide-se nas seguintes fases:

1. Formulação da Pergunta de Pesquisa

2. Seleção das Bases de Dados

3. Seleção dos Estudos

4. Extração de Informação

5. Resumo dos Resultados

\subsection{Literatura sobre Repositórios de Objeto de Aprendizagem}

Ao realizar uma pesquisa da literatura já existente relativa aos ROAs, foram encontrados vários estudos. McGreal (2008) classifica os repositórios em três categorias: os que fornecem o conteúdo que armazenam; os que fornecem link para recursos hospedados em outros locais; e os que funcionam de forma híbrida, fornecendo recursos por eles armazenados e links para outras bases. Neste mesmo estudo, foram reveladas quais são as principais funcionalidades de interação com um repositório. Tzikopoulos, Manouselis e Vuorikari (2009) analisaram quais as principais características de 59 populares ROAs. No trabalho de Ochoa e Duval (2009) foi apurado o tamanho dos repositórios e as suas taxas médias de crescimento e de publicação de conteúdo. Também foram encontrados vários estudos que objetivam integrar ROAs a Ambientes Virtuais de Aprendizagem (AVAs), como evidenciado em Schmitt et al. (2013) e Tarouco, Rodrigues e Schmitt (2013). Zervas, Alifragkis e Sampson (2014) fizeram uma análise quantitativa das funcionalidades oferecidas por estes sistemas. E, por fim, Clements, Pawlowski e Manouselis (2015) investigaram abordagens de qualidade para este tipo de repositório. 
Contudo, não foi encontrado nenhum estudo que apresentasse detalhes de estratégias utilizadas para a realização do Controle de Versão dos recursos contidos nesses repositórios. Afim de investigar este tópico, esta RS tem como objetivo responder a seguinte pergunta de pesquisa:

- Como é realizado o Controle de Versão de Objetos de Aprendizagem em Repositórios de Objeto de Aprendizagem que possuem esta característica?

\subsection{Protocolo de Revisão}

O Protocolo de Revisão elaborado tem como objetivo selecionar publicações que descrevem detalhes sobre como é realizado o versionamento de Objetos de Aprendizagem em um ROA, já que a pergunta de pesquisa só pode ser respondida por meio da descrição do funcionamento e das peculiaridades das estratégias de Controle de Versão.

No mês de Maio de 2015, realizou-se consultas nos seguintes repositórios internacionais de publicações a que os autores tinham acesso e que foram elencados em Brereton et al. (2007) e em EBSE Project (2007) como bases digitais relevantes para a área da Engenharia de Software: IEEExplorer; ACM Digital Library; Google Scholar; CiteSeerX; ScienceDirect; SpringerLink; e SCOPUS. A essa lista, também foi considerado interessante adicionar o serviço indexador Web of Science.

As strings de consulta utilizadas em cada repositório foram elaboradas a partir da seguinte String Genérica:

- Abstract ("Learning Object Repository" OR "Learning Resource Repository" OR "Learning Content Repository" OR "Learning Object Database" OR "Learning Resource Database" OR "Learning Content Database") AND ("Version Control" OR "Revision Control" OR "Change Control" OR "Version Management" OR "Change Management" OR "Versioning")

Basicamente, o objetivo é que sejam retornadas publicações que possuam em seus resumos o termo "Learning Object Repository" ou um dos seus sinônimos descritos e também possuam em algum lugar do texto o termo "Version Control" ou uma das expressões relacionadas. Ademais, é importante ressaltar que apenas foram consideradas publicações realizadas nos últimos 10 anos, mais precisamente de 2005 até o momento da execução do protocolo.

As strings de pesquisa retornaram um total de 299 publicações. A Tabela 1 apresenta a string utilizada em cada repositório, conforme as suas especificidades, juntamente com o quantitativo de publicações obtido.

Tabela 1 - Consultas realizadas nos repositórios

\begin{tabular}{|l|l|c|}
\hline Repositório & \multicolumn{1}{c|}{ String de Consulta } & Qtd. \\
\hline IEEE & $\begin{array}{l}\text { ("Abstract": "Learning Object Repository" OR "Learning Resource Repository" OR } \\
\text { "Learning Content Repository" OR "Learning Object Database" OR "Learning Resource } \\
\text { Database" OR "Learning Content Database") AND ("Version Control" OR "Revision Con- } \\
\text { trol" OR "Change Control") } \\
\text { Publication Year: 2005 - 2015 } \\
\text { Obs.: pelo fato da ferramenta de pesquisa limitar o número de termos de consulta, foram } \\
\text { realizadas duas execuções. Primeiramente a segunda cláusula foi ("Version Control" OR } \\
\text { "Revision Control" OR "Change Control"), em seguida foi ("Version Management" OR } \\
\text { "Change Management" OR "Versioning"). }\end{array}$ & 3 \\
\hline $\begin{array}{l}\text { ACM Digital } \\
\text { Library }\end{array}$ & $\begin{array}{l}\text { (Abstract: "Learning Object Repository" or Abstract: "Learning Resource Repository" or } \\
\text { Abstract: "Learning Content Repository" or Abstract: "Learning Object Database" or }\end{array}$ & 0 \\
\hline
\end{tabular}

V. $14 \mathrm{~N}^{\mathrm{o}}$ 2, dezembro, 2016 


\begin{tabular}{|c|c|c|}
\hline & $\begin{array}{l}\text { Abstract: "Learning Resource Database" or Abstract: "Learning Content Database" ) and ( } \\
\text { "Version Control" or "Revision Control" or "Change Control" or "Version Management" } \\
\text { or "Change Management" or "Versioning" ) } \\
\text { Published since: } 2005\end{array}$ & \\
\hline $\begin{array}{l}\text { Google } \\
\text { Scholar }\end{array}$ & $\begin{array}{l}\text { Com a frase exata: "Version Control" } \\
\text { Com no mínimo uma das palavras: "Learning Object Repository" "Learning Resource } \\
\text { Repository" "Learning Content Repository" "Learning Object Database" "Learning } \\
\text { Resource Database" "Learning Content Database" } \\
\text { Onde minhas palavras ocorrem: Em qualquer lugar do artigo } \\
\text { Exibir artigos com data entre: } 2005 \text { - } 2015 \\
\text { Não incluir patentes / Não incluir citações. } \\
\text { Obs.: por limitações da ferramenta de busca, foram realizadas seis consultas distintas. Para } \\
\text { cada consulta foi utilizado um valor diferente para o campo "Com a frase exata". }\end{array}$ & 281 \\
\hline CiteSeerX & $\begin{array}{l}\text { abstract:("Learning Object Repository" OR "Learning Resource Repository" OR "Learning } \\
\text { Content Repository" "Learning Object Database" OR "Learning Resource Database" OR } \\
\text { "Learning Con-tent Database") AND ("Version Control" OR "Revision Control" OR } \\
\text { "Change Control" OR "Version Management" OR "Revision Management" OR "Change } \\
\text { Management" OR "Versioning") AND year:[2005 TO 2015] }\end{array}$ & 10 \\
\hline ScienceDirect & $\begin{array}{l}\text { ABSTRACT("Learning Object Repository" OR "Learning Resource Repository" OR } \\
\text { "Learning Content Repository" OR "Learning Object Database" OR "Learning Resource } \\
\text { Database" OR "Learning Content Database") AND ("Version Control" OR "Revision Con- } \\
\text { trol" OR "Change Control" OR "Version Management" OR "Change Management" OR } \\
\text { "Versioning") } \\
\text { Publication Date: } 2005 \text { - Present }\end{array}$ & 0 \\
\hline SpringerLink & $\begin{array}{l}\text { (ab:("Learning Object Repository") OR ab:("Learning Resource Repository") OR } \\
\text { ab:("Learning Content Repository") OR ab:("Learning Object Database") OR } \\
\text { ab:("Learning Resource Database") OR ab:("Learning Content Database")) AND } \\
\text { ("Version Control" OR "Revision Control" OR "Change Control" OR "Version } \\
\text { Management" OR "Change Management" OR "Versioning") } \\
\text { Date Published: } 2005 \text { - } 2015 \\
\text { Don’t Include Preview-Only Content }\end{array}$ & 3 \\
\hline SCOPUS & $\begin{array}{l}\text { ABS ( "Learning Object Repository" OR "Learning Resource Repository" OR "Learning } \\
\text { Content Repository" OR "Learning Object Database" OR "Learning Resource Database" } \\
\text { OR "Learning Content Database" ) AND ALL ( "Version Control" OR "Revision Control" } \\
\text { OR "Change Control" OR "Version Management" OR "Change Management" OR } \\
\text { "Versioning" ) AND PUBYEAR > } 2005\end{array}$ & 2 \\
\hline $\begin{array}{l}\text { Web of } \\
\text { Science }\end{array}$ & $\begin{array}{l}\text { TS=("Learning Object Repository" OR "Learning Resource Repository" OR "Learning } \\
\text { Content Repository" OR "Learning Object Database" OR "Learning Resource Database" } \\
\text { OR "Learning Con-tent Database") AND TS=("Version Control" OR "Revision Control" } \\
\text { OR "Change Control" OR "Version Management" OR "Change Management" OR } \\
\text { "Versioning") } \\
\text { Tempo estipulado = } 2005-2015 \\
\text { Idioma da pesquisa = Auto } \\
\text { Obs.: a ferramenta de pesquisa não permite a busca de termos em todo o texto da } \\
\text { publicação nem somente no seu resumo, mas apenas em alguns elementos textuais } \\
\text { específicos. Por este motivo, optou-se por fazer a pesquisa pelo elemento Tópico (TS). }\end{array}$ & 0 \\
\hline
\end{tabular}

As publicações obtidas foram avaliadas para determinar quais de fato auxiliariam a responder à pergunta de pesquisa da RS. Para isso, elas devem respeitar os seguintes critérios para que sejam consideradas na fase de Extração de Informações:

(i) Corresponder de fato à String Genérica de consulta. A limitação técnica de algumas ferramentas possibilita que sejam retornadas publicações em desacordo com a intenção de pesquisa da RS. Por exemplo, os repositórios Google Scholar e Web of Science não autorizam a consulta por termos especificamente nos resumos das 
publicações, isto pode resultar no retorno de publicações que não possuem no resumo o termo "Learning Object Repository" ou uma de suas variantes.

(ii) Ser um artigo na língua inglesa.

(iii) Ter sido publicado em um Jornal, Revista ou Anais de Conferência.

(iv) Descrever, em detalhes, o Controle de Versão de Objetos de Aprendizagem em, no mínimo, um Repositório de Objeto de Aprendizagem.

Como uma primeira triagem das 299 publicações encontradas, foi verificada a correspondência com a String Genérica de consulta, se eram artigos em inglês e onde foram publicadas, ou seja, os três primeiros critérios de aceitação.

Tabela 2 - Quantitativo de publicações excluídas na primeira triagem

\begin{tabular}{|l|c|}
\hline \multicolumn{1}{|c|}{ Motivo } & Quantidade \\
\hline Por não corresponder com a String Genérica de consulta & 116 \\
\hline Por não ser um artigo (Livros, Teses, Relatórios, dentre outros) & 117 \\
\hline Por não ser um artigo em inglês & 7 \\
\hline Por não possuir acesso na íntegra ao conteúdo da publicação & 16 \\
\hline Por terem sido retornadas em mais de um repositório & $\mathbf{2 6 1}$ \\
\hline \multicolumn{1}{|c|}{ Total } & 5 \\
\hline
\end{tabular}

Ressalto que, dos 38 artigos selecionados na primeira triagem, 12 foram aceitos mesmo não atendendo à primeira cláusula da String Genérica, que exige a presença de algum dos sinônimos do termo "Learning Object Repository" no resumo. Eles foram aceitos pelo fato de tratarem, em algum nível, do Controle de Versão de recursos.

Posteriormente, foi executada, com as publicações remanescentes da primeira triagem, uma análise mais apurada. Os resumos dos artigos foram lidos com o objetivo de averiguar se o conteúdo da publicação aborda detalhes referentes às funcionalidades ou mesmo questões técnicas de, no mínimo, um Repositório de Objeto de Aprendizagem, ou se apresenta uma proposta para funcionamento e/ou organização de conteúdo nestes tipos de bases digitais. Esta verificação resultou na exclusão de 19 artigos.

Por fim, numa terceira avaliação, foi realizada a leitura da introdução e conclusão dos artigos, objetivando detectar se a publicação retrata de forma detalhada como se dá o versionamento de recursos em ROAs.

Após a execução de todo esse Protocolo de Revisão, 6 artigos foram selecionados para serem lidos por completo, de modo a avaliar se realmente descrevem a forma, com nível de detalhe, de como é feito o versionamento de OAs em ROAs. Desses 6, apenas 2 detalham estratégias de Controle de Versão.

\section{Discussão e Dados Extraídos}

\subsection{Lehmann, Hildebrandt, Rensing e Steinmetz}

Lehmann, Hildebrandt, Rensing e Steinmetz, em "Capture, management, and utilization of lifecycle information for learning resources", publicado no Journal IEEE Transactions on Learning Technologies, em 2008, propuseram uma arquitetura distribuída com o objetivo de capturar, processar, gerenciar e utilizar informações produzidas ao longo do ciclo de vida de um OA. Para isso, também foi proposta uma extensão para o padrão de metadados LOM. Esta extensão visa possibilitar o armazenamento de informações 
relacionadas ao ciclo de vida do recurso, mais precisamente, as alterações que ocorrerem nos metadados que o descrevem.

Segundo os autores, as informações relacionadas ao ciclo de vida de um RED são um tipo especial de metadado que refere-se a um processo. Na publicação, é descrito que a vida de um OA é composta pelas fases de: Autoria; Distribuição; Utilização; e Reautoria. Porém, em muitos casos, os dados gerados nesta última fase e a interligação da versão nova com a antiga não são consideradas. Os autores apresentam uma série de cenários em que essas informações podem ser proveitosamente utilizadas.

As informações geradas ao longo do ciclo de vida de um OA podem referir-se a dois pontos: aos relacionamentos e ao contexto. Sendo que as de contexto são específicas de um recurso e as de relacionamento diz respeito à conexão de recursos. Inclusive, na publicação, os autores apresentam quais são os tipos de relações possíveis de se estabelecer entre OAs. Dentre eles, são citados o Relacionamento de Versão, que ocorre quando um recurso é a correção ou atualização de outro, e o Relacionamento de Variação, que ocorre quando um recurso é a adaptação de outro a um novo contexto.

O padrão de metadados LOM foi escolhido por Lehmann, Hildebrandt, Rensing e Steinmetz para ser estendido, porque é um padrão amplamente difundido e utilizado para descrever OAs. Das categorias desse padrão, os autores concentraram-se nas: $2-\mathrm{Li}$ fecycle; 3 - Meta-Metadata; e 7 - Relation. Porém, como o principal objetivo era armazenar relacionamentos dentro do padrão LOM, os autores escolheram a Categoria 7 (Relation) para a expansão, já que ela é destinada ao armazenamento de informações sobre relacionamentos.

Os autores expandiram a Categoria 7 com a criação do campo Changes, que objetiva armazenar o que mudou nos metadados do recurso. Ele é um campo composto por um ponteiro para o campo alterado, pelo valor anterior do campo e pelo momento em que ocorreu a alteração. Além disso, eles descreveram no artigo um vocabulário próprio a ser utilizado na Categoria 7 para descrever cada tipo de relacionamento que pode ocorrer entre OAs. No caso de um Relacionamento de Versão, usa-se hasVersion ou isVersionOf; já no caso de um Relacionamento de Variação, hasVariant ou isVariantOf. A Figura 1 apresenta um exemplo dessa expansão contido na publicação (utilizou-se $o$ Relacionamento de Redução - isReductionOf).

Figura 1 - Exemplo de extensão da Categoria 7 (Relation) do padrão LOM

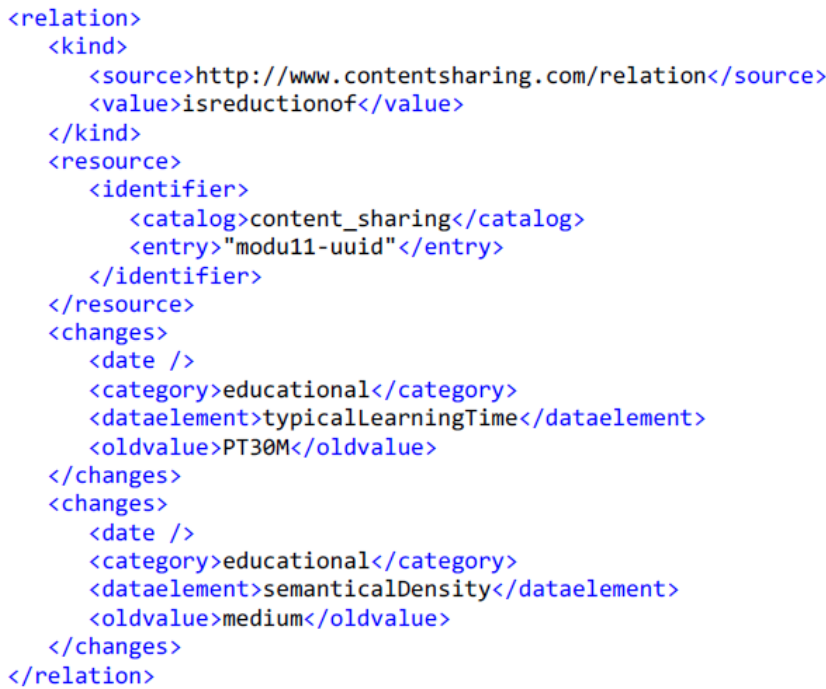


A estratégia apresentada na publicação permite manter o histórico de mudanças entre diferentes versões de um recurso e pode ser utilizada para qualquer tipo de relação, mas, em especial, é útil para os Relacionamentos de Versão e de Variação, pois armazena a sintática das mudanças feitas, tornando possível a automatização do Controle de Versão.

\subsection{Libbrecht e Brooks}

Libbrecht e Brooks, em "Publication of Distributed Linked Content", publicado nos Anais do First Doctoral Consortium in Technology Enhanced Learning, em 2006, investigaram como REDs podem ser produzidos e publicados de forma distribuída. Para isso, eles precisaram analisar como gerenciar os links entre partes do conteúdo dos recursos e os seus respectivos metadados, como também a evolução destes elementos. Nesse trabalho, também foi apurada a noção de produções derivadas, ou seja, versões diferentes de um mesmo recurso, e descrita a forma como é realizado o gerenciamento de versão por ferramentas de versionamento de artefatos de software.

Os estudos foram realizados com base na experiência em produção de conteúdo obtida com o ActiveMath, um ambiente web de aprendizagem de matemática, e com o iHelp Courses, um sistema web para o gerenciamento de OAs. Na publicação, são apresentadas diferentes estratégias para a reutilização de recursos nestes tipos de ambientes, dentre elas, a de utilizar Links Externos para referenciar outros REDs. Porém, é destacado que, neste caso, é importante garantir confiabilidade e disponibilidade, já que o autor de um conteúdo não consegue ter o total controle sobre todos os recursos referenciados. É afirmado que com o IMS Content Packaging (IMS Global Learning Consortium, 2016) é possível reutilizar OAs através dessa estratégia, pois ele permite o estabelecimento de referências.

Em relação às produções derivadas, é afirmado no artigo que manter um link entre o recurso original e a sua versão derivada é importante para o gerenciamento, pois permite ao autor da nova versão adotar melhorias à medida que o autor da versão original as realiza, possibilita o rastreio de como o conteúdo de um recurso é modificado ao longo do tempo e facilita a localização de suas extensões ou de suas versões corrigidas. Com isso, os autores propõem duas estratégias para o versionamento de recursos em ambientes distribuídos para autoria de OAs. Uma para o ActiveMath e outra destinada a repositórios baseados em padrões, categoria em que o iHelp Courses se enquadra. Porém, apenas na descrição da segunda estratégia é que se apresentam detalhes referentes ao Controle de Versão.

A segunda estratégia propõe a utilização de uma ferramenta de autoria que possibilita o gerenciamento de diferentes versões de um recurso, o Learning Object Versioning Environment (LOVE). O LOVE é fruto da pesquisa de mestrado do segundo autor do artigo. Em seu estudo, Brooks destacou que uma estratégia de versionamento de OAs deve preservar o histórico de evolução de conteúdo e levar em consideração o estado da arte dos padrões voltados para o e-learning. Para tanto, ele elaborou o LOVE para a manutenção do histórico de modificação dos recursos, armazenando a sintática das alterações, e também para a visualização simplificada dos relacionamentos entre versões. 
Figura 2 - Tela da Ferramenta LOVE

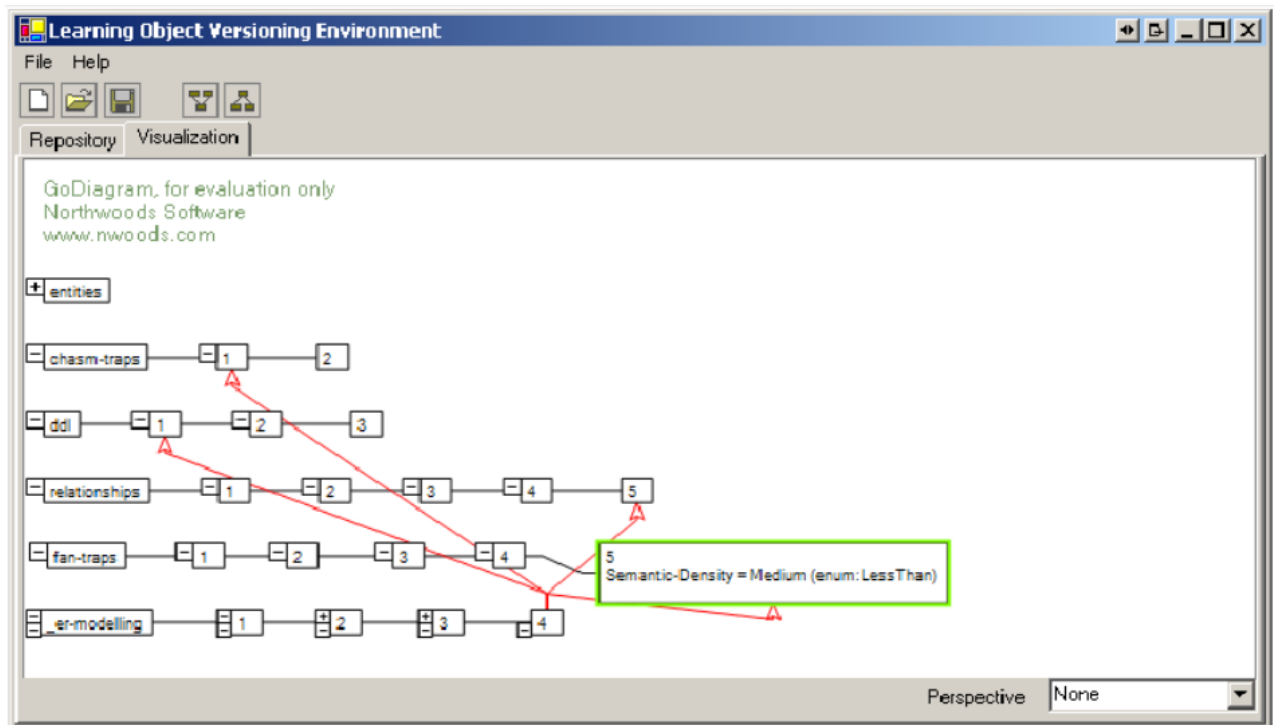

Essa ferramenta utiliza o padrão IMS Content Packaging (IMS Global Learning Consortium, 2016). Ela embute as modificações que originaram a versão do OA no IMS Content Packaging Manifest do recurso. Manipulações de árvore realizadas na árvore de atividade IMS Simple Sequencing também são adicionadas ao pacote de conteúdo que descreve todo o conteúdo do OA. Esta maneira de preservar as alterações torna a criação de produções derivadas tão simples quanto copiar o pacote original e editá-lo.

Dentre as limitações dessa estratégia de versionamento estão os fatos de as alterações em uma versão, como uma correção de erro, e a inclusão de novos metadados não se propagarem para as demais versões. Porém, o último caso é considerado positivo por Brooks, já que o metadado é algo intrinsicamente ligado a uma versão do recurso, não sendo possível estimar o impacto da sua modificação nas demais versões (Brooks, 2005).

\section{Conclusão}

Foi evidenciado ao longo da Revisão Sistemática que é comum a produção de diversas versões de um Objeto de Aprendizagem, tanto para corrigir erros ou realizar atualizações quanto para adaptar os recursos a outros contextos de uso, tornando necessário que os repositórios deste tipo de RED disponham de estratégias para o versionamento de recursos.

Esta RS objetivou levantar quais são essas estratégias de gerenciamento de versões, de modo a auxiliar equipes responsáveis pela modelagem e implementação deste tipo de repositório durante o processo de decisão sobre como realizar o versionamento dos recursos. A pergunta de pesquisa indicou este objetivo:

- Como é realizado o Controle de Versão de Objetos de Aprendizagem em Repositórios de Objeto de Aprendizagem que possuem esta característica?

Para responder à pergunta, oito bases internacionais de publicações relevantes para a área da Engenharia de Software foram consultadas. As primeiras pesquisas retornaram um total de 299 publicações. Foi verificado quais delas ajudavam a responder à pergunta de pesquisa, conforme os critérios especificados no Protocolo de Revisão. Como resultado das análises, 6 artigos foram selecionados para serem lidos integralmente, mas, após a leitura, foi detectado que apenas 2 respondiam à pergunta. As 
estratégias de versionamento de OAs apresentadas nestas publicações foram descritas na seção Discussão e Dados Extraídos.

A revisão permite concluir que o Controle de Versão dos recursos depositados em um ROA é basicamente realizado através de duas estratégias: estendendo e/ou alterando os padrões de metadados comumente utilizados para a descrição de OAs ou adaptando o modelo de dados para a persistência de recursos nos repositórios. Os dois métodos buscam permitir o estabelecimento de relações entre os recursos, de um modo que garanta a identificação do tipo de relacionamento existente entre as versões e a preservação dos aspectos que as diferenciam, informações necessárias para o efetivo gerenciamento de versões. Inclusive, das duas publicações selecionadas por meio do Protocolo de Revisão, em uma o versionamento é feito através de uma extensão no padrão de metadados LOM enquanto que na outra é utilizada uma ferramenta de autoria para a acomodação de informações de controle na própria estrutura dos OAs, para que sejam mantidos relacionamentos entre eles quando persistidos em repositórios.

\section{Referências Bibliográficas}

BIOLCHINI, J. et al. Systematic Review in Software Engineering. Rio de Janeiro: [s.n.], mai. 2005. Universidade Federal do Rio de Janeiro (Technical Report ES 679/05)

BRERETON, P. et al. Lessons from Applying the Systematic Literature Review Process within the Software Engineering Domain. Journal of Systems and Software. v.80, n.4, p.571-583, 2007.

BROOKS, C. A. H. Supporting Learning Object Versioning. Dissertação (Mestrado em Ciência da Computação) - University of Saskatchewan, Saskatoon, 2005.

CASTELLS, M. A sociedade em rede. 8. ed. São Paulo: Paz e Terra, 1999.

CISCO SYSTEMS. Reusable learning object strategy: Designing and developing learning objects for multiple learning approaches. [S.1.]: [s.n.], 2003. 34p. CISCO (White Paper)

CLEMENTS, K.; PAWLOWSKI, J.; MANOUSELIS, N. Open educational resources repositories literature review - Towards a comprehensive quality approaches framework. Computers in Human Behavior, v.51, p1098-1106, oct. 2015.

EBSE PROJECT. Guidelines for Performing Systematic Literature Reviews in Software Engineering. Keele: [s.n.]; Durham: [s.n.], jul. 2007. v. 2.3. (EBSE Technical Report)

FARRELL, S. (Ed.). A Guide to Web Preservation. 2010. Disponível em: < https://jiscpowr.jiscinvolve.org/wp/files/2010/06/Guide-2010-final.pdf >. Acesso em: 28 out. 2016.

HIGGS, P. E.; MEREDITH, S.; HAND, T. Technology for Sharing: Researching Learning Objects and Digital Rights Management. Hobart: [s.n.], 2003. Australian National Training Authority (Flexible Learning Leader Report 2002)

IMS GLOBAL LEARNING CONSORTIUM. IMS Content Packaging Specification, v1.1.4. Disponível em: < https://www.imsglobal.org/content/packaging/index.html\#version1.1.4 >. Acesso em: 30 oct. 2016.

KITCHENHAM, B. Procedures for Performing Systematic Reviews. Keele: [s.n.]; Eversleigh: [s.n.], jul. 2004. Keele University (Technical Report TR/SE-0401); Australian Technology Park (NICTA Technical Report 0400011T.1)

LEHMANN, L. et al. Capture, Management, and Utilization of Lifecycle Information for Learning Resources. IEEE Transactions on Learning Technologies. v.1, n.1, p.75-87, jan. 2008. 
LIBBRECHT, P.; BROOKS, C. Publication of Distributed Linked Content. In: Doctoral Consortium in Technology Enhanced Learning, 1., 2006, Crete. Proceedings. Crete: [s.n.], p.21-32, 2006.

MCGREAL, R. A Typology of Learning Object Repositories. In: ADELSBERG, Heimo et al. (ed.). Handbook on information technologies for education and training. 2 ed. [S.l.]: Springer, 2008. Cap. 1. p.5-28.

OCHOA, X.; DUVAL, E. Quantitative Analysis of Learning Object Repositories. IEEE Transactions on Learning Technologies. v.2, n.3, p.226-238, 2009.

RENSING, C. et al. Re-Use, Re-Authoring, and Re-Purposing of Learning Resources Definitions and Examples. Darmstadt: [s.n.], 2005. 16 p. (KOM-TR-2005-02).

SCHMITT, M. A. R. et al. Depósito de objetos de aprendizagem em repositórios a partir da integração com ambientes virtuais de aprendizagem. Revista Novas Tecnologias na Educação, Porto Alegre, v.11, n.3, dez. 2013.

SRIDHARAN, B.; DENG, H.; CORBITT, B. Critical success factors in e-learning ecosystems: a qualitative study. Journal of Systems and Information Technology. v.12, n.4, p. 263-288, 2010.

TAROUCO, L. M. R. et al. Objetos de Aprendizagem: teoria e prática. Porto Alegre: Evangraf, 2014. $504 \mathrm{p}$.

TAROUCO, L. M. R.; RODRIGUES, A. P.; SCHMITT, M. A. R. Integração do MOODLE com repositório abertos. Revista Perspectivas em Ciência da Informação, Belo Horizonte, v.18, n.1, p.66-85, jan./mar. 2013.

TAROUCO, L. M. R.; SILVA, C. C. G.; GRANDO, A. Fatores que afetam o reuso de objetos de aprendizagem. Revista Novas Tecnologias na Educação, Porto Alegre, v.9, n.1, jul. 2011.

TATE, M.; HOSHEK, D. A Model for the Effective Management of Re-Usable Learning Objects (RLOs): Lessons from a Case Study. Interdisciplinary Journal of E-Learning and Learning Objects. v.5, n.1, p.51-72, 2009.

THEILMANN, W.; ALTENHOFEN, M. Versioning of E-Learning Objects Enabling Flexible Reuse. In: IADIS International Conference on WWW/Internet, 2003, Algarve. Proceedings. Algarve: [s.n.], p.719-727, 2003.

TZIKOPOULOS, A.; MANOUSELIS, N.; VUORIKARI, R. An Overview of Learning Object Repositories. In: HALPIN, Terry (ed.). Selected Readings on Database Technologies and Applications. Hershey: Igi Global, 2009. Cap. 3. p.44-64.

WILEY, D. A. Learning Object Design and Sequencing Theory. Tese (Doutorado em Filosofia) - Brigham Young University, Provo, 2000.

ZERVAS, P.; ALIFRAGKIS, C; SAMPSON, D. G. A Quantitative Analysis of Learning Object Repositories as Knowledge Management Systems. Knowledge Management \& E-Learning. Hong Kong, v.6, n.2, p.156-170, 2014. 